\title{
Caetano da Rosa, Catarina: Androiden als Spie(ge)l der Aufklärung, 184 S., Avinus, Hamburg 2020.
}

\section{Christian Vater}

Angenommen: 6. Juli 2021 / Online publiziert: 23. Juli 2021

(C) Der/die Autor(en) 2021

Dieses kluge und detailscharfe Buch erscheint als Überblicksdarstellung genau zur richtigen Zeit, um der rezenten und brisanten Debatte um Künstliche Intelligenz im deutschsprachigen Raum historiografische Tiefenschärfe zu geben. Wenn sich in der Gegenwart, auch in Reaktion auf die Geltungsansprüche der futurologischen Meistererzählungen des silicon valley, die Forderung nach einer ,Digitalen Aufklärung ‘ gesellschaftlich aufdrängt, bietet sich ein Blick auf den Umgang mit Technologie in der Zeit der ersten Aufklärung an. Als kulturwissenschaftliche Publikation passt das Ergebnis fachlich gut neben Jessica Riskins „The Restless Clock“ (2016) (die aus der Perspektive der Geschichte der Lebenswissenschaften schreibt) und lässt sich durch Adrienne Mayors „Gods and Robots“ (2018) ergänzen, in dem diese Erzählung bis in die klassische Antike verlängert wird.

Bereits ein erster Blick in das lobenswert feinteilige Inhaltsverzeichnis zeigt den Wert des vorliegenden Bandes. Sortiert in die Kategorien „Philosophie“, „Technik“ und „Literatur“ stellt die Verfasserin Androiden in das Zentrum ihrer Darstellung der Zeit der Aufklärung. Als epistemisches Leitartefakt entwickelt sich entlang derer Eigenarten das Denken, wobei der „Automat als Ideal“ gesetzt und zum Gedankenexperiment genutzt wird. Die Entwicklung der mechanischen Künste auf höchstes handwerkliches Niveau kann mit Blick auf „Androiden in der Werkstatt“ technikhistorisch nacherzählt werden. Als Motiv und Bildgeber regen „Androiden als Subjekte“ darüber hinaus die Phantasie an, und somit die schöne Literatur auch als ,Schauerromantik‘. Um Androiden als Idee und Artefakt werden die Gedanken und Werke der Größen der Zeit gruppiert: René Descartes, Gottfried Wilhelm Leibniz, Thomas Hobbes und Julien Offray de La Mettrie in der Philosophie (unerwartet ergänzt durch Friedrich II. und den unbekannten Johann Karl Wezel); in der

Christian Vater $(\square)$

KIT, Karlsruhe, Deutschland

E-Mail: christian.vater@partner.kit.edu 
mechanischen Kunst Jacques de Vaucanson, Friedrich von Knauss, Wolfgang von Kempelen und Pierre Jaquet-Droz; in der Literatur Jean Paul, Heinrich von Kleist, E.T.A. Hoffmann und (bemerkenswert) Georg Büchner.

Das Ziel des kulturwissenschaftlichen Ansatzes liest sich wie ein Aufruf zur ,Wissensarchäologie“ in Museum, Archiv und Magazin: „Kunstobjekte müssen aktualisiert werden, um lebendige Existenz zu erlangen. Oft sind die Meisterwerke zerstört, verbrannt oder verschollen. Was bleibt sind nur verstaubte Reste eines staunenswerten Erfindungsreichtums. Diese Studie setzt sich zum Ziel, die narrative Dimension von nur teilweise erhaltenen Museumsstücken zu erschließen“ (S. 17). Dieses Vorgehen erlaubt nun aber auch einen weitergreifenden Erkenntnisgewinn: Die „Funktion“ der Androiden für das 18. Jahrhundert kann durch die Rekonstruktion der „,Sprache der Objekte““ „,entschlüsselt“" werden (S. 17). Die Uhrwerksartefakte, die so ins Zentrum der Aufmerksamkeit rücken, sind so vielfältig wie faszinierend: Von Knauss’ „Schreibende Wundermaschine“, Vaucassons „Querflötist“" oder von Kempelens „Schachtürke“ zeigen dies schon in einer exemplarischen Aufreihung.

Eine Frage, die nach der Lektüre bleibt, ist, ob Androiden nicht nur der Spiegel (oder ein Spiel) der Aufklärung seien - und das sind sie bestimmt - sondern auch, ob wir uns in diesen Uhrwerksautomaten noch immer selbst begegnen (können) oder ob vielleicht der black mirror des Smartphones in der Gegenwart diese Funktion übernommen hat. Wenn wir Menschen Maschinen (gewesen) sein sollen, dann schließt sich außerdem die Frage an: Maschinen welcher Art? Kraftmaschinen, Rechenmaschinen und Schreibmaschinen würden ja durchaus unterschiedlich zu konstruieren sein und Anlass zu verschiedenartigen Vergleichen bieten. In diese Maschinentypen wäre auch ein je anderer Zweck hineingelegt worden als in Unterhaltungsmaschinen, die im Gegensatz zu Werkzeugen der Hand- oder Kopfarbeit ja ludische und durchaus auch repräsentative Funktionen hätten erfüllen müssen. Das Uhrwerk als ,Leittechnologie “ und anthropologischer Bildgeber der Aufklärung bietet ausgehend vom vorliegenden Band jedenfalls einen belastbaren Startpunkt für eine technisch interessierte historische Epistemologie. Am Ende der Studie kann dann auch festgehalten werden: „Die These der Mensch-Maschine beschreibt verschiedene Zusammenhänge auf metaphorische Weise. Sie ähnelt einem Proteus, gleicht einer mathematischen List und entspricht einer geometrischen Methode: Sie ist der Versuch, eine Ordnung zu beschreiben und zu schaffen, die sich von einem Fach auf ein anderes übertragen lässt"“ (S. 131).

Der Band ist reich verzettelt (das Taschenbuch hat auf 128 Textseiten 843 Fußnoten) und bietet 20 Abbildungen, von denen einige (auch online) schwer greifbar sind. Als Index dient ein Personenregister. Ein Vorwort der Berliner Nietzsche-Forscherin Renate Reschke verknüpft die historische Betrachtung kenntnisreich mit Fragen der Gegenwart.

Ein ausführliches Verzeichnis von Quellen (leider nicht vollständig historischkritisch bibliografiert) sowie eine umfangreiche, historisch tiefe und sehr nützliche Sammlung von Forschungsliteratur runden den Band ab - hier vermisst man vielleicht Gaby Woods „Living Dolls“ (2002), das oben bereits genannte Buch von Riskin und den Katalog zur von Kempelen-Ausstellung im Karlsruher Zentrum für Kunst und Medien (ZKM) „Mensch-[in der]-Maschine“, herausgegeben von Bern- 
hard Serexhe und Peter Weibel (2007). Der Aufsatz „,Du lebloses, verdammtes Automat!'. Unser Schicksal vis-à-vis Maschinen und Automaten ..." des ausgewiesenen Descartes-Experten Hans-Peter Schütt zur ideengeschichtlichen Vertiefung des Automaten-Motivs kam sicherlich zu spät, um Berücksichtigung zu finden. Was man sich abschließend für den gehobenen Preis hätte wünschen können, wäre ein besserer Bilddruck gewesen - wenn nicht auch ein gut gebundenes Hardcover für dieses wunderbare Buch eine bessere Form geboten hätte. Der Band gewann den Förderpreis der (ehemaligen) Deutschen Gesellschaft für Geschichte der Medizin, Naturwissenschaft und Technik (DGGMNT) und nach der anregenden Lektüre bleibt festzuhalten: zu Recht.

Funding Open Access funding enabled and organized by Projekt DEAL.

Open Access Dieser Artikel wird unter der Creative Commons Namensnennung 4.0 International Lizenz veröffentlicht, welche die Nutzung, Vervielfältigung, Bearbeitung, Verbreitung und Wiedergabe in jeglichem Medium und Format erlaubt, sofern Sie den/die ursprünglichen Autor(en) und die Quelle ordnungsgemäß nennen, einen Link zur Creative Commons Lizenz beifügen und angeben, ob Änderungen vorgenommen wurden.

Die in diesem Artikel enthaltenen Bilder und sonstiges Drittmaterial unterliegen ebenfalls der genannten Creative Commons Lizenz, sofern sich aus der Abbildungslegende nichts anderes ergibt. Sofern das betreffende Material nicht unter der genannten Creative Commons Lizenz steht und die betreffende Handlung nicht nach gesetzlichen Vorschriften erlaubt ist, ist für die oben aufgeführten Weiterverwendungen des Materials die Einwilligung des jeweiligen Rechteinhabers einzuholen.

Weitere Details zur Lizenz entnehmen Sie bitte der Lizenzinformation auf http://creativecommons.org/ licenses/by/4.0/deed.de.

\section{Todd, David: A Velvet Empire. French Informal Imperialism in the Nineteenth Century, 368 S., Princeton UP, Princeton, NJ u.a. 2021.}

\section{Jürgen Osterhammel}

Angenommen: 30. Juni 2021 / Online publiziert: 4. August 2021

(C) Der/die Autor(en) 2021

Auch in der buchbetonten Geschichtswissenschaft können gelegentlich Aufsätze klassischen Rang erreichen. 1953 veröffentlichten zwei junge Dozenten, John Gallagher und Ronald Robinson, später einflussreiche Professoren in Cambridge und

\footnotetext{
Jürgen Osterhammel $(\varangle)$

Albert-Ludwigs-Universität Freiburg im Breisgau, Freiburg i. Br., Deutschland

E-Mail: juergen.osterhammel@ frias.uni-freiburg.de
} 